\title{
SOXHLET-RANDALL METHOD MODIFICATION FOR RAPID LIPID EXTRACTION ON EXAMPLE OF RAINBOW TROUT
}

\author{
Margarita Kochneva $^{1}$, Leonid Butusov ${ }^{1,2}$, Sergey Bindukov ${ }^{1}$, Vasiliy Ivlev ${ }^{1}$ \\ ${ }^{1}$ Peoples' Friendship University of Russia, Russia; \\ ${ }^{2}$ Prokhorov General Physics Institute of the Russian Academy of Sciences, Russia \\ kochneva-mv@rudn.ru, leonid.butusov@ya.ru, sergeyang@mail.ru,chemistron@mail.ru
}

\begin{abstract}
The study examines lipid extraction using a modification of the Soxhlet-Randall method. The essence of the modification is based on the improvement of such key performance indicators of the method as the extraction degree and its time, which also were boosted by avoiding fat hydrolysis stage using a binary solvent mixture - chloroform and ethanol in different ratios (1:1, 1:2, 1:4). As an example, rainbow trout (Oncorhynchus mykiss) bred on a fish farm was chosen for practical study, which reduced the likelihood of contaminants in the fish and therefore ensured the maximum purity of the experiment. With the help of an automatic extractor, a modification of the Soxhlet-Randall method was carried out for qualitative and quantitative characteristics. The optimal intervals for the three stages of the extraction of fats - immersion, extraction and washing. After the first extraction, the thimble with the sample rises up and is washed with a condensable mixture of solvents, i.e. the solvents are reused, which is an additional advantage of the method. Studies have been carried out on the subject of residual protein content by the Kjeldahl method, which did not exceed $3 \%$, while the amino-acid profile of the protein residue was not considered. The dry residue from the thimble of the automatic extractor was additionally tested by the Soxhlet arbitration method used in assessing the quality of fat in the food industry and showed $95 \%$ completeness of fat extraction from trout with a rainbow modified method. The modification of the Soxhlet method has good prospects for use in the food industry to assess the quality of fish products, but it requires additional research using the example of various fish species to establish correction factors.
\end{abstract}

Keywords: lipid, Soxhlet, extraction, rainbow trout.

\section{Introduction}

The fast-growing rates of aquaculture reproduction of salmon due to the high content of polyunsaturated fatty acids (an average of 2.3 grams per $100 \mathrm{~g}$ fillets) increase the interest of scientists and stimulate new researches on the chemical composition and the development of new methods for efficient extraction. Polyunsaturated fatty acids are classified as essential nutritional factors, and their content should be constantly from 4 to $6 \%$ of the energy value. It is very important that the ratio of PUFAs of the $\omega-6$ and $\omega-3$ families in the diet of a healthy person is 10: 1, and in cases of pathology of lipid metabolism $-5: 1$ or even 3: 1 . In this regard, the definition of a quality indicator, such as fat content, is especially important in the development of laboratory equipment to reduce the time for preparation, extraction and subsequent analysis.

Raw fat is obtained in various ways (thermal, hydromechanical, enzymatic, fat freezing, etc.), which are based on the principle of destruction of the structure of adipose tissue and the separation of fat from it. Fat in the raw materials is enclosed in fat cells, which are organically linked by intercellular substance consisting of collagen and elastic fibers. Inside the cells, fat and other components form complex colloidal systems that must be destroyed to release fat.

Fish oil extraction processes can be classified into three categories: physical, which includes heating, pressing and filtering; biological - enzymatic oil extractions; chemical solvent extraction [1].

Chemical extraction can be classified by few methods - Bligh Dyer, Soxhlet, accelerated extraction and extraction on supercritical fluids, as well as modifications of these methods and a number of less popular methods using microwave radiation, acids and alkalis, membranes, etc. [2].

The standard method of Bligh Dyer involves the use of methanol, chloroform and water as solvents, the method is well studied, allows determining the total amount of lipids and analyzing the samples without pre-drying. At the same time, it is rather labor-consuming and takes a lot of time due to the long time separation of the extraction mixture and additional equipment, such as a vacuum pump, rotary evaporator, homogenizer, is also required [3].

The Soxhlet method uses various organic solvents, such as hexane, acetone, ether, toluene, etc., it is quite simple, halogen-free solvents could be used, which is important for the environment, it could be automated. The main disadvantage is the need for hydrolysis, which significantly increases the time spent on extraction. 
The method of accelerated solvent extraction is based on the use of high temperature and pressure, which reduces the extraction time and the amount of the solvent used, but this method is significantly more expensive than the above [4].

Supercritical extraction using carbon dioxide can be used to extract substances that are thermally unstable or prone to oxidation, which refers to lipids. The method does not use organic solvents, what makes its undoubted advantage, has a high extraction rate, but with all the benefits it is incredibly expensive [5].

Due to the fact that aquatic biological resources are distinguished by a large variety in the chemical structure of lipids, there are many aspects of extraction. It determines the possibility of improvements for the existing extraction methods and creation of new ones, which are meant for certain species of fish, such as discussed in this article salmon species.

In this research we consider different modifications of the Soxhlet method using as the example of fish a rainbow trout (Oncorhynchus mykiss) with the use of automatic extraction setup, which in addition to automating the process can significantly reduce the consumption of the amount of the solvent, but at the same time has only general recommendations for extraction using a modified Soxhlet method.

\section{Materials and methods}

Rainbow trout from a fish farm was used in the research. Pre-defrosted to $-1^{\circ} \mathrm{C}$ (in the thick) fish was purified from mechanical impurities, butchered, removed all the entrails with caviar or milt, the spine, ribs and skin removed.

Filet was milled in a meat grinder until smooth. Samples of the resulting mass were taken and dried in an oven at $105{ }^{\circ} \mathrm{C}$ for 120 minutes. Drying stage is necessary for Soxhlet methods to remove moisture. Samples of $5 \pm 0.1 \mathrm{~g}$ were used for the Soxhlet method as an optimal for raw materials with fat content from 6 to $10 \%$, what is governed by the manual for the automatic extractor and also because 5 grams of the test substance completely occupies the working surface of the extraction sleeve.

Arbitrage method of Bligh Dyer [6] was performed manually and because it does not need drying, the results of extraction were recalculated by the equation (1) for correct comparison with Soxhlet automatic extraction methods.

$$
m=\frac{T 2-T 1}{S W} \times 100,
$$

where $m$-amount of extracted lipids $(\%)$;

$T 2$ - glass weight before extraction;

$T 1$ - glass weight after extraction;

$S W$ - weight of the sample.

Soxhlet methods with dried raw fat were performed using different solvents and their ratios with an automatic fat extractor VELP SER 148/6 under different temperature. The list of the methods with abbreviations and the result information are presented in Table 1.

Methods $A$ \& $G$ as standard methods for manual and automatic extraction were iterated 3 times, methods B-F were iterated 5 times. Average values are shown in the results table (Table 1).

NMR spectra of the extracted samples were measured by Jeol JNM ECA 600 and processed by Mnova NMR Software.

\section{Results and discussion}

Different ratios of ethanol-chloroform mixtures, as well as different temperatures at which fat will be extracted, were used during the experiment to determine the optimal method (methods a-f). Because the research is aimed at automatic extraction, the recommended method for automatic extractors was also prepared using diethyl ether. The results of the study are shown in Table 1.

Method (a) used in Russia as an arbitration method [7] showed the lipid content of $7.5 \%$. The residual substances after extraction were examined for the protein content $(21.8 \%)$, moisture 
$(68.88 \%)$ and ash (1.5\%), what in total gives $99.68 \%$ and indicates the complete extraction of lipids using the control method. In our study, we proceeded from the results of this method, taking them as the reference value.

In view of possible discrepancies in the amount of extracted lipids, 5 extractions were performed for each of the methods; Table 1 shows the average values of the extracted lipid relative to the sample in percent. We noted that for methods, which use ethanol in some cases, we observe a decrease in reproducibility; if for the Bligh Dyer method the amount of extracted lipids differed by no more than $0.1 \%$, which in turn can be attributed to weight errors of the sample on the stage of preparation, using ethanol these discrepancies were up to $3 \%$ relative to each other.

Table 1

\section{Extraction methods}

\begin{tabular}{|c|c|c|c|c|c|}
\hline $\begin{array}{c}\text { Sample } \\
\text { name }\end{array}$ & Solvent & Method & Ratio & $\begin{array}{c}\text { Extraction } \\
\text { temperature, }{ }^{\mathbf{0}} \mathbf{C}\end{array}$ & $\begin{array}{c}\text { Average } \\
\text { quantity of } \\
\text { lipid, \% }\end{array}$ \\
\hline $\mathrm{A}$ & $\begin{array}{c}\mathrm{C}_{2} \mathrm{H}_{5} \mathrm{OH}, \\
\mathrm{CH}_{3} \mathrm{Cl}, \mathrm{H}_{2} \mathrm{O}\end{array}$ & Bligh and Dyer & 1 & 23 & 7.5 \\
\hline $\mathrm{B}$ & $\mathrm{CH}_{3} \mathrm{Cl}$ & Soxhlet & 1 & 160 & 4.48 \\
\hline $\mathrm{C}$ & $\begin{array}{c}\mathrm{C}_{2} \mathrm{H}_{5} \mathrm{OH} \\
\mathrm{CH}_{3} \mathrm{Cl}\end{array}$ & Soxhlet & $1: 1$ & 210 & 9.45 \\
\hline $\mathrm{D}$ & $\begin{array}{c}\mathrm{C}_{2} \mathrm{H}_{5} \mathrm{OH} \\
\mathrm{CH}_{3} \mathrm{Cl}\end{array}$ & Soxhlet & $1: 1$ & 160 & 9.65 \\
\hline $\mathrm{E}$ & $\begin{array}{c}\mathrm{C}_{2} \mathrm{H}_{5} \mathrm{OH} \\
\mathrm{CH}_{3} \mathrm{Cl}\end{array}$ & Soxhlet & $1: 2$ & 210 & 7.6 \\
\hline $\mathrm{F}$ & $\begin{array}{c}\mathrm{C}_{2} \mathrm{H}_{5} \mathrm{OH} \\
\mathrm{CH}_{3} \mathrm{Cl}\end{array}$ & Soxhlet & $1: 4$ & 160 & 7.88 \\
\hline $\mathrm{G}$ & $\mathrm{Et}_{2} \mathrm{O}$ & Soxhlet & 1 & 110 & 6.84 \\
\hline
\end{tabular}

At the same time, we eliminate the measurement error associated with the initial mass of the sample, due to the characteristics of the Soxhlet method, in which wiping of glass used for the sample with a cotton wool wetted in a solvent with the subsequent transfer to the extractor's sleeve is provided.

According to Table 1, the samples obtained by the Soxhlet method using the ethanol-chloroform solvent combination in a $1: 2$ ratio at an extraction temperature of $210{ }^{\circ} \mathrm{C}(\mathrm{e}), 1: 4$ at $160{ }^{\circ} \mathrm{C}(\mathrm{f})$, are the closest to the arbitration method (a), as well as the extract obtained using diethyl ether as a solvent ( $\mathrm{g}$ ).

For sample (b), we observed solid formations, which are usually associated with a complex of chemical processes of lipo-amine condensation and formation of melanoidins, which explains the small yield percentage of the method. Increasing the temperature of the reaction mixture also affects the formation of flocculent inclusions, i.e. accelerates polymerization.

Figure 1 shows the changes of the extracted samples colors, so the Bligh Dyer method (a) as well as Soxhlet extraction with diethyl ether have orange colors, caused by the presence of the pigment Astaxanthin inherent in the studied fish and extracts obtained by binary mixtures are quite dark because of the polymerization process.

Extraction with chloroform-ethanol mixtures at temperatures below $160{ }^{\circ} \mathrm{C}$ was ineffective due to incomplete evaporation during the sample drying step. In turn, increasing the amounts of ethanol results in the need to increase the extraction temperature, because active ethanol evaporation occurs directly at $210^{\circ} \mathrm{C}$.

Extraction by the classical method for the automatic extractor using diethyl ether showed incomplete extraction of lipids (6.84\% vs. $7.5 \%$ as etalon value), which confirms the theory that binary solvents [8] for this kind of products are necessary. 
Samples obtained by the arbitration method (a), the classical method for automatic extraction (g), as well as by Soxhlet with use of binary mixtures of chloroform and ethyl alcohol in a ratio of 1: 4 (f) and 1: 2 (e) have been investigated for nuclear magnetic resonance (Fig. 2).



Fig. 1. Colors of extracted samples F, E, G and A

The spectra of the samples obtained by the methods (f, e, d) are identical to the spectrum of the arbitration method using lipid signals, but there is also an additional signal at $3.36 \mathrm{ppm}$. Method (e) is characterized by the presence of an additional quartet signal of ethanol $(3.72 \mathrm{ppm})$ and a signal not related to the fat fraction $(3.22 \mathrm{ppm})$, which is not explained in this paper.

The data obtained confirm the need to increase the extraction temperature when using ethanol in a binary mixture of solvents in an amount greater than $25 \%$. At the same time, an increase of the extraction temperature, as mentioned above, can increase the reactivity of the mixture and cause the formation of melanoidins and, therefore, reduce the extraction yield.

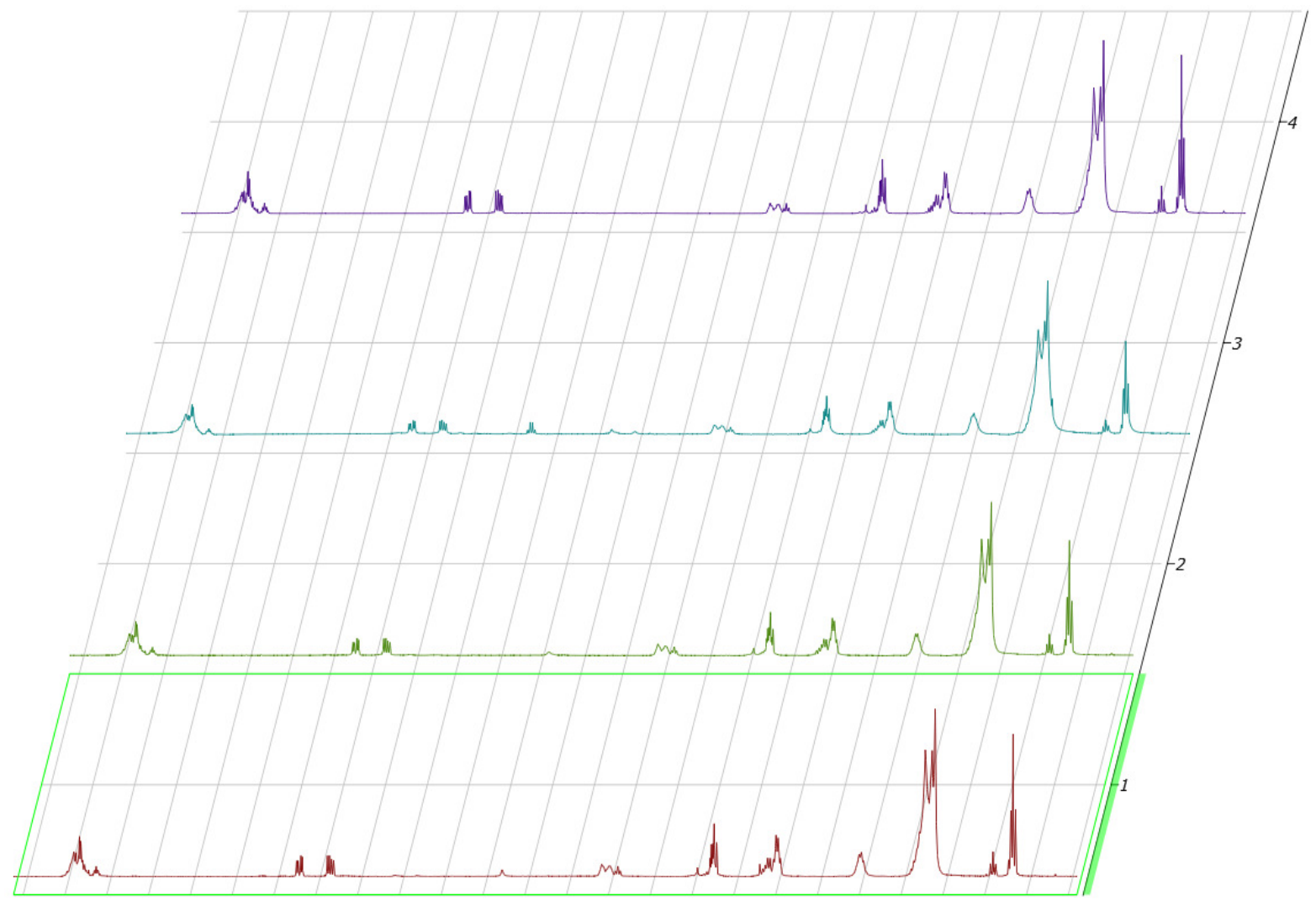



Fig. 2. NMR stack spectra of samples F (1), E (2), G (3), A (4)

Nevertheless, the obtained results are very important, since the use of a binary mixture of solvents allows us to avoid the stage of hydrolysis and, accordingly, significantly reduce the time of extraction. Due to the lack of standards for automatic extraction, the extraction time requires careful selection for each type of fish. In our work it was a total of 2 hours: 30 minutes of immersion in boiling solvent, 60 minutes of irrigation flushing time and 30 minutes of solvent recovery time. For a binary mixture, a preliminary stage of hydrolysis of the sample is not required; this significantly reduces the extraction 
time up to 3 hours. At the same time, such time savings require detailed consideration of the interaction with the sample and the solvent mixture, the search for their optimal ratio and temperature extraction mode, as it was done in this study.

According to the results of our study, a modification of the Soxhlet method for automatic extractor using a binary solvent mixture of ethanol - chloroform in a 1:4 ratio has a good convergence with the Bligh Dyer's arbitration method $-7.6 \%$ vs. $7.5 \%$ of extracted lipids and more effective than recommended by the manufacturer Soxhlet method with the use of diethyl ether, which gives $6.84 \%$ of fat.

These results could be recommended for manufacturers of such devices for inclusion in the techniques and also for modification of extractors by the use of more resistant materials with the assumption that they are reproducible for salmon species.

\section{Conclusions}

Extraction of intracellular lipids from rainbow trout was investigated using an automatic extractor with a binary mixture of ethanol-chloroform solvents; different ratios alcohol - chloroform were tested as well as a comparison with the methods of Bligh and Dyer method and Soxhlet extraction with diethyl ether was given.

It was established that for a 1:4 ratio of ethanol-chloroform mixture, the extraction temperature should be within 160 , and increasing the proportion of ethanol to 50 percent or more requires an increase in the extraction temperature to 210.

Comparison of the lipid samples obtained using binary mixtures of solvents and the samples obtained by extraction using the arbitrary method of Bligh Dyer and Soxhlet using diethyl ether was carried out, beginning of lipoamine polymerization with increasing the extraction temperature was shown.

NMR spectroscopy shows the relationship of increasing the mass of the extract with increasing concentration of ethanol in the solvent mixture.

The modified method is significantly more effective than the method recommended by the manufacturer $-7.6 \%$ vs. $6.84 \%$ and is comparable to the arbitration method with the hydrolysis stage $-7.5 \%$.

\section{Acknowledgements}

This publication was prepared with the support of the "RUDN University Program 5-100".

\section{References}

[1] Adeoti I.A., Hawboldt K. A review of lipid extraction from fish processing by-product for use as a biofuel. Biomass and Bioenergy, pp. 330-339.

[2] Ivanovs K., Blumberga D.. Extraction of fish oil using green extraction methods: a short review. Energy Procedia, vol. 128, 2017, pp. 477-483

[3] Bligh, E.G. and Dyer, W.J. A rapid method for total lipid extraction and purification. Canadian Journal Biochemistry and Physiology, vol 37, 1959, Canada pp. 911-917

[4] Mottaleb, M. A., Sarker S. D.. Accelerated Solvent Extraction for Natural Products Isolation. Natural Products Isolation, vol. 864, 2012, pp. 75-87.

[5] Rubio-Rodríguez, N. de Diego, Beltrán S. M., Jaime, I. S., Sanz M. T. Supercritical fluid extraction of fish oil from fish by-products: A comparison with other extraction methods. Journal of Food Engineering, vol. 109(2). 2012, pp. 238-248

[6] Bligh E. G., W. J. Dyer. A rapid method of total lipid extraction and purification. Canadian Journal of Biochemistry and Physiology, 1959, 37(8): 911-917

[7] GOST 7636-85. "Fish, marine mammals, invertebrates and products of their processing. Methods of analysis (with change №1)" (In Russian).

[8] Biondo P. B. F., et al.. A new method for lipid extraction using low-toxicity solvents developed for canola (Brassica napus L.) and soybean (Glycine max L. Merrill) seeds Analytical Methods, vol. 7, 2015, pp. 9773-9778. 